\title{
The Biosurveillance Resource Directory - A One-Stop Shop for Systems, Sources, and Tools
}

\author{
Kristen Margevicius*, Mac Brown, Lauren Castro, William B. Daniel, Eric N. Generous, \\ Kirsten Taylor-McCabe and Alina Deshpande
}

Los Alamos National Laboratory, Los Alamos, NM, USA

\section{Objective}

The goal of this project is to identify systems and data streams relevant for infectious disease biosurveillance. This effort is part of a larger project evaluating existing and potential data streams for use in local, national, and international infectious disease surveillance systems with the intent of developing tools to provide decision-makers with timely information to predict, prepare for, and mitigate the spread of disease.

\section{Introduction}

Local, national, and global infectious disease surveillance systems have been implemented to meet the demands of monitoring, detecting, and reporting disease outbreaks and prevalence. Varying surveillance goals and geographic reach have led to multiple and disparate systems, each using unique combinations of data streams to meet surveillance criteria. In order to assess the utility and effectiveness of different data streams for global disease surveillance, a comprehensive survey of current human, animal, plant, and marine surveillance systems and data streams was undertaken. Information regarding surveillance systems and data streams has been (and continues to be) systematically culled from websites, peer-reviewed literature, government documents, and subject-matter expert consultations.

\section{Methods}

A relational database has been developed and refined to allow for detailed analyses of data streams and surveillance systems. To maximize the utility of the database and facilitate one-stop-shopping for biosurveillance system information, we have expanded our scope to include not only biosurveillance systems, but also data sources, tools, and biosurveillance collectives. Captured in the information collected about the resource (if available) is the name and acronym of the resource, the date the resource became available, the accessibility of the resource (is it open to all, or are there limitations to access), the primary sponsors, if the resource is associated with GIS functionality, and if the focus is health. Also collected is contact information, information regarding the scope and domain of the resource, the pertinent diseases or disease categories, and the geographic and pop- ulation coverage of the resource. Websites associated with the resource are directly accessible from the database. Data stream information is also captured based on our developed data stream framework. If the resource uses other specified systems/sources/tools for data gathering or analysis, then that is also captured and directly linked within the database.

\section{Results}

The Biosurveillance Resource Directory (BRD) is in the process of being tested by multiple potential end users in the public health, biosecurity, and biosurveillance communities. Feedback from these testers is being used to refine the database to maximize functionality and utility. Additionally, methods for dynamically updating and maintaining the database are being evaluated. Automated and semi-automated queriable reports have been developed and are integral to demonstrating specific use-case scenarios in which the BRD would be beneficial for end-users.

\section{Conclusions}

A need for a biosurveillance one-stop shop has been increasingly called for to help in evaluating what data streams and systems are available and relevant for many different biosurveillance needs and goals. The prototype Biosurveillance Resource Directory is a searchable, dynamic database for biosurveillance systems, sources, and tools information.

\section{Keywords}

infectious disease; biosurveillance; database

\section{Acknowledgments}

This project is supported by the Chemical and Biological Technologies Directorate Joint Science and Technology Office (JSTO), Defense Threat Reduction Agency (DTRA).

*Kristen Margevicius

E-mail: kmargevicius@lanl.gov 